\title{
On the representation of units by cyclotomic: polynomials
}

by

Varkmo EnNota (Turki)

Let $m$ he a naturel number, $\Phi_{m}(x)$ the $m$ th cyclotomic polynomial, and $\zeta=\zeta_{m}$ a suitably chosen primitive $m$ th root of unity. By a $J$-field we mean an algebraic number field which is either totally real or a totally imaginary quadratic extension of a totally real field. For the general theory of such fields we refer the reader to [4]. Clearly abelian extensions of $Q$ (i.e. subfields of cyclotomic fields) are $J$-fields. Subfields, joins, and normal closures of $J$-fields are álso $J$-fields.

Let $K$ be a $J$-field. By $S_{\infty}$ we denote the set of archimedean valuations of $K$. Following Györy [4], we call a non-archimedean valuation $\psi$ of $K$ real iff $\psi(\alpha)==\psi(\bar{\alpha})$ for all $\alpha \in K$, where $\bar{\alpha}$ denotes the complex conjugate of $\alpha$. If $K_{0}$ denotes the maximal real subfield of $K$, then $\psi$ is real if and only if $p$ is either ramified or inert in the extension $K / K_{0}$. Let $S$ be a finite set of valuations of $K$ containing $S_{\infty}$. An element $\alpha$ of $K$ is called an $S$-integer (S-unit) iff $\psi(\alpha) \leqslant 1(\psi(\alpha)=1$ ) for all valuations $\psi$ of $K$. not containod in $S$.

Let $p>3$ be a prime and put $K=Q\left(\zeta_{p_{2}}\right)$. Grossman [3] has investigated the diophantine equation

$$
\Phi_{m i}(\xi)=\eta
$$

where $\xi$ and $\eta$ are units of $T$ and $\xi$ is not a root of unity. He showed that the aquation (1) has no solntions if $m>2, m \neq 3,6$, and determined the solutions for $m=3$ or 6 . To further noted that if $K$ is any $J$-field, $\eta$ is a tunit of $K$, and $\xi$ is a non-zero number of $K$ which is not a root of unity, then from the validity of $(1)$ it follows that $m \leqslant m_{0}$ for some cons tant $m_{0}$ deponding on the degree [K: $\left.Q\right]$. From the results of Schinzel ([7], cf. also [6]) it follows that the sarne holds for any field $K$. Grossman asks whether $m_{0}$ may be found independent of $[K: Q]$ in the case when $K$ is a $J$-field. The lollowing theorem answers this question in the affirmative.

THWOMU .1. Let $\xi$ be a non-zero algebraio number not a root of unity such that $Q(\xi)$ is a of field. Suppose that $m \geqslant 5, m \neq 6$, and that $\Phi_{m}(\xi)$ 
is an $S$-unit for some finite set $S \supseteq S_{\infty}$ of valuations of the field $Q(\xi, \zeta)$ such that every $\psi \in S \backslash S_{\infty}$ is real. Then $Q(\xi)$ is an abelian extension of $Q$ and $m \leqslant m_{0}$ for some absolute constant $m_{0}$.

From the proof it should be easy to obtain a numerical value for $m_{0}$. One should also be able to find all possible solutions for the remaining values of $m$ as in [3]. A natural question is what can be said in the case when $S$ is a set of valuations of $Q(\xi)$ and not of $Q(\xi, \zeta)$. Obviously the the orem does not remain true in this generality. This is seen trivially by thiking $\xi$ totally real. Less trivial examples are the following oncs. Thalke $\alpha=3+\sqrt{3}, \xi=\sqrt{-\alpha}$. Then $K=Q(\xi)$ is a non-normal extension of $Q$ which is a $J$-field. We have

$$
\begin{aligned}
& \Phi_{8}(\xi)=\xi^{4}+1=a^{2}+1=13+6 \sqrt{3}, \\
& \Phi_{16}(\xi)=\xi^{8}+1=a^{4}+1=253+144 \cdot \sqrt{3}, \\
& \Phi_{32}(\xi)=\xi^{16}+1=a^{8}+1=7(17959+10368 \sqrt{3}) .
\end{aligned}
$$

Let $S^{\prime}$ denote the set of primes of $K$ lying above $13+6 \sqrt{3}, 253+1444 \sqrt{3}, 7$, $17959+10368 \sqrt{3}$, and put $S=S_{\infty} \cup S^{\prime}$. It is not hard to check that the primes in $S^{\prime}$ are real.

In connection with the result of Schinzel it is also natural to consider representations by the homogenized cyclotomic polynomial $\Phi_{m}(x, y)$ $=y^{q(m)} \Phi_{m}(x / y)$. In this case we can prove

THEOREM 2. Let $\xi$ and $\eta$ be non-zero algebraic numbers sueh that $Q(\xi, \eta)$ is a J-field. Let $S \supseteq S_{\infty}$ be a finite set of valuations of the field $Q(\xi, \eta, \xi)$ such that every. $\psi \in S \backslash S_{\infty}$ is real. Suppose that $\xi$ and $\eta$ are S-integers and that $\Phi_{m}(\xi, \eta)$ is an S-unit. Then either $\bar{\eta} \mid \xi$ is a root of unity or there exist absolute constants $m_{1}<m_{2}$ such that $m \leqslant m_{2}$ and for $m \geqslant m_{1} Q(\xi|\xi, \eta| \xi, \vec{\eta} \mid \xi)$ is an abelian extension of $Q$.

In the case when $\bar{\eta} \mid \xi$ is a root of nnity, writing $\bar{\eta}=\xi \theta^{2}, \omega=\eta \theta$, we have $\Phi_{m}(\bar{\omega}, \omega)=\theta^{q(m)} \Phi_{m}(\xi, \eta)$. It is easy to construct examples where $\omega$ is an $S$-unit for a suitable $S$ showing that there is no absolute bound, for $m$, but excluding this ease the question still remains open whether there then is such a bound.

Finally, taking into account the close connection between tho nature of $\Phi_{m}(\xi, \eta)$ and the existence of a primitive divisor of the expression $\xi^{n h}-\eta^{n n}$ (see [6] and [7]), one is led to ask whether there exists and absolutie constant $m_{0}$ such that the expression $\xi^{n}-\eta^{m}$ has a primitive divisor for $m \geqslant m_{0}$. when $(\xi, \eta)=1, \xi / \eta$ is not a root of unity, and $Q(\xi, \eta)$ is a $J$-field.

Proof of the theorems. We shall use an idea of Newman. (c.. [2]) combined with results of Györy [4] and the recent results of Conway and Jones $[1]$ on relations between roots of unity. Wo bogin with a proof of the first part of Theorem 1. Put $\varepsilon_{k}=\xi-\zeta^{k}((k, m)=1)$. Let $\psi$ be any valuation of the field $Q(\xi, \zeta)$ which does not belong to $S$. Since $\psi\left(\Phi_{m}(\xi)\right)$ $=1$ and $\Phi_{m}(x)$ is a monic polynomial with integral coefficients, we have $\psi(\xi) \leqslant 1$. Therefore clearly $\psi\left(\varepsilon_{k}\right)=1$ for each $k$, so that the $\varepsilon_{k}$ are $S$-units. From [4], p. 154, Th. 1, it follows that $\varrho_{k}=\bar{\varepsilon}_{k} / \varepsilon_{k}$ is a root of unity for each \%. From the assumption of our theorem $\varphi(m) \geqslant 3$. Therefore it is possible to choose $\zeta$ so that $\varepsilon_{1}$ is not purely imaginary. From the equations $\tilde{\varepsilon}_{1}=\varrho_{1} \varepsilon_{1}, \vec{\varepsilon}_{-1}=\varrho_{-1} \varepsilon_{m-1}$ we obtain

$$
\begin{aligned}
\left(\varrho_{1}-\varrho_{-1}\right) \varepsilon_{1} & =\left(1+\varrho_{-1}\right)\left(\zeta-\zeta^{-1}\right), \\
\left(\varrho_{1}-\varrho_{-1}\right) \varepsilon_{-1} & =\left(1+\varrho_{1}\right)\left(\zeta-\zeta^{-1}\right) .
\end{aligned}
$$

Since $\varepsilon_{1}$ is not purely imaginary, $\varrho_{1} \neq-1$, whence $\varrho_{1} \neq \varrho_{-1}$. Thus $\varepsilon_{1}$ belongs to a cyclotomic field so that $Q(\xi)$ is an abelian extension of $Q$. This proves the first parti of Thcorem 1. The second part is clearly contained in Theorem 2, whence in what follows we shall only be concerned with the proof of that.

As above we now denote $\varrho_{l k}=\left(\xi-\zeta^{-k} \bar{\eta}\right) /\left(\xi-\zeta^{k} \eta\right)$ for any $k$ with $(7, m)=1$, where again the $e_{k}$ are roots of unity. We assume that $\bar{\eta} / \xi$ is not a root of unity otherwise there is nothing to prove. We always suppose that $m$ is sufficiently large. Talko any $r$ with $(r ; m)=1, r \neq \equiv \pm 1$ mod $m$. Wo have

$$
\begin{aligned}
\xi-\zeta^{-1} \bar{\eta}-\varrho_{1}(\xi-\zeta \eta) & =0, \\
\xi-\zeta \bar{\eta}-\varrho_{-1}\left(\xi-\zeta^{-1} \eta\right) & =0, \\
\xi-\zeta^{-n} \bar{\eta}-\varrho_{r}\left(\xi-\zeta^{r} \eta\right) & =0, \\
\xi-\zeta^{r} \bar{\eta}-\varrho_{-r}\left(\xi-\zeta^{-r} \eta\right) & =0 .
\end{aligned}
$$

Consider (3) as a system of homogeneous linear equations in the unknowns $\xi, \vec{\eta}, \xi, \eta$. The determinant of the coefficient matrix

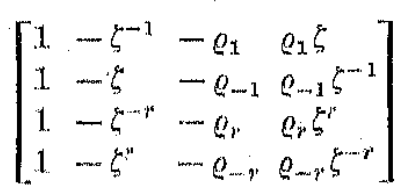

must vanish. Computing the detrorminant wo obtain

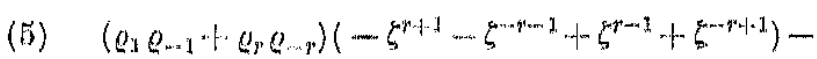

$$
\begin{aligned}
& -\varrho_{1} \varrho_{r}\left(\zeta^{2 r}-2 \zeta^{r+1}+\zeta^{2}\right)-\varrho_{-1} \varrho_{-r}\left(\zeta^{2 r}-2 \zeta^{-r-1}+\zeta^{-2}\right)+ \\
& +\varrho_{1} \varrho_{-r}\left(\zeta^{-2 r}-2 \zeta^{-r+1}+\zeta^{2}\right)+\varrho_{-1} \varrho_{r}\left(\zeta^{2 r}-2 \zeta^{r-1}+\zeta^{-2}\right)=0 .
\end{aligned}
$$

Suppose that the rank of $(4)$ is less than three. Then in particular the ledding three by three minor determinant of (4) vanishes so that we have

$$
\left(\zeta-\zeta^{-1}\right) \varrho_{r}+\left(\zeta^{-1}-\zeta^{-r}\right) \varrho_{-1}+\left(\zeta^{-r}-\zeta\right) \varrho_{1}=0
$$


Using the language of Conway and Jones [1.], let $T$ denoto the vanishing formal sum of roots of unity corresponding to the left-hand side of $(6)$. Consider the root $\zeta_{Q_{r}}$. If $T$ does not involve $\zeta_{r}$, then there is another term on the left-hand side of (6) equal to $\angle \zeta_{Q_{r}}$, and these two terms cancel out. On the other hand, if $T$ does involve $\zeta Q_{r}$, then there is a minimal out. On the other hand, involving $\zeta \varrho_{r}$. From [1], p. 235, Th. 5, it follows that the reduced exponent of $T_{1}$ divides 30 . Thus in both esuses there is another term on the left-hand side of $(6)$ equal to $a \zeta_{e}$, where $a^{310}=1$. This term eannot be $-\zeta^{-1} e_{r}$ if $m$ is large enough. Hence we have an equation of the form

$$
a \zeta Q_{r}=\zeta^{-a r-b} \varrho_{u} \quad(a \in\{0,1\} ; b \in\{0, \pm 1\} ; u \in\{ \pm 1\}) .
$$

Writing $X=\zeta^{r}$ we obtain from (3)

$$
\xi X-\bar{\eta}-a^{-1} \xi^{-1-b} \varrho_{u} X^{1-a}(\xi-\eta X)=0 .
$$

Consider (7) as an equation in the unknown $X$. Since $\ddot{\eta} / \xi$ is not a root of unity, (7) does not reduce to an identity. As there are only a linite number of equations (7), we obtain a contradiction for $m$ sufficiently large choosing $r$ suitably. Hence the rank of (4) must be three, so that all the ratios of $\xi, \bar{\eta}, \xi, \eta$ belong to a cyclotomio field. This proves the second assertion of Theorem 2 .

Before proceeding further with the proof of Theorem. 2 we need the following tions

LFinM. Let $\xi$ and $\eta$ be non-zero complex numbers satisfying the condi-

$$
\xi=\tau \xi, \quad \bar{\eta}=\tau \eta, \quad \xi-\zeta^{-k} \bar{\eta}=\varrho_{k}\left(\xi-\zeta^{k} \eta\right) \quad(k \in Z,(\hbar, m)=1)
$$

where $\tau$ and the $\varrho_{1}$ are roots of unity. Then either $\xi= \pm \eta$ or $m \leqslant m_{3}$ for some absolute constant $m_{3}$.

Proof. Determine $\theta_{l k}$ so that $\theta_{k}^{*}=\varrho_{k} \tau^{-1}$. For ench $k$ with $(k, m)=1$ we then have

$$
\xi / \eta=\left(\theta_{k} \zeta^{k}-\theta_{k_{k}}^{-1} \zeta^{k i}\right) /\left(\theta_{k}-\theta_{k}^{-1}\right),
$$

and in particular we see that $\theta_{k}^{2} \neq 1, \zeta^{-2 h}$. Cornbining the eggation. (8) as it stands and. with 7 replaced by 1 wo get the relation

$$
\text { (9) } \begin{aligned}
-\theta_{1} \theta_{k} \zeta+\theta_{1} \theta_{k}^{-1} \zeta & +\theta_{1} \theta_{k} \zeta^{k}-\theta_{1}^{-1} \theta_{k} \zeta^{k}- \\
& -\theta_{1}^{-1} \theta_{k}^{-1} \zeta^{-1}+\theta_{1}^{-1} \theta_{k} \zeta^{-1}+\theta_{1}^{-1} \theta_{k i}^{-1} \zeta^{-k}-\theta_{1} \theta_{k}^{-1} \zeta^{-10}=0 .
\end{aligned}
$$

We suppose that $m>m_{3}$ for a sufficiently large absolute constant $m_{\mathrm{s}}$ and show that then $\xi= \pm \eta$. Arguing as before we obtain a non-trivial equation of the form

$$
\text { (10) } \quad a \theta_{1} \theta_{k} \zeta=\theta_{1}^{a} \theta_{k}^{b} \zeta^{c k+d} \quad\left(a, b \in\{ \pm 1\} ; a, d \in\{0, \pm 1\} ; a^{210}=1\right) \text {. }
$$

Consider first the possibility $b=1$. Then we must have $c=0$, otherwise we obtain a contradiction choosing $k^{\prime}$ suitably which is possible for large enough $m$. From (9) we now find that the only possibility is $a=d$ $=-1$, whenee $\theta_{1}^{2}=a^{-1} \zeta^{-2}$. From (8) with $k \approx 1$ we get

$$
\xi / \eta=(1-\alpha) \zeta /\left(1-\alpha \zeta^{2}\right),
$$

and we have a contradiction by assuming that $\zeta$ was originally so chosen that this equation does not hold for any $a$ with $\alpha^{210}=1$.

Thus $b=-1$. Jut $X:=\zeta^{k}$. Combining (8) and (10) we obtain

$$
(\xi / \eta)\left(a^{-1} 0_{1}^{a-1} \zeta^{d-1} X^{a+1}-X\right)-a^{-1} 0_{1}^{a-1} \zeta^{l-1} X^{c+2}+1=0 .
$$

Since this equation must reduce to an identity, we easily find that $c=-1$ and $\xi / \eta= \pm 1$. This proves the lemma.

Consider now the relation (5). Again we have a non-trivial equation

(11) $\left.\quad a \varrho_{2} \varrho_{-1} \zeta^{r+1}=\varrho_{u} \varrho_{v}\right\}^{(a r+b)} \quad(u ; v \in\{ \pm 1, \pm r\} ; a, b \in\{0, \pm 1, \pm 2\} ;$

$$
\left.\alpha^{M}=1\right) \text {, }
$$

where $A T$ denotes the product of primes $<20$. Wo cannot have $\varrho_{u} \varrho_{v}=\varrho_{1} \varrho_{-1}$ otherwise we get a contradiction for a suitable $r$. (Note that if $a=1$ then $b=-1$, which is impossible for large $m$.)

Suppose first that $\varrho_{u} \varrho_{v}=\varrho_{r} \varrho_{-r}$. Write $X=\zeta^{r}, \tau^{2}=\alpha \varrho_{1} \varrho_{-1} \zeta^{1-b}$ so that $\varrho_{r} \varrho_{-n}=\tau^{2} X^{1-a}$. From (3) we have

$$
(\xi X-\bar{\eta})(\xi-\bar{\eta} X)=r^{2} X^{1-a}(\xi X-\eta)(\xi-\eta X) .
$$

This equation must reduce to an identity, whonee necessarbily $a=1$ and

$$
\xi^{2}+\eta^{2}=\tau^{2}\left(\xi^{2}+\eta^{2}\right), \quad \overline{\xi \eta}=\tau^{2} \xi \eta .
$$

Hence $(\xi+\eta)^{2}=\tau^{2}(\xi+\eta)^{2}$. Choosing tho sign of $\tau$ suitably we thus have either $\xi=\tau \xi, \ddot{\eta}=\tau \eta$ or $\xi=\tau \eta, \bar{\eta}=\ldots \tau$. However, from the lemma we infer that in both canses $\eta / \xi$ is an root of unity, a contradiction.

Suppose next that $u= \pm$. I and $v=r$. Then $a \in\{0,1,2\}$. Writing $X=\zeta^{r}$ Wo have from (11) $e_{r}=\alpha \varrho_{\ldots 1} \zeta^{1-b)} X^{1-a}$, and then (3) implies

$$
\xi X-\eta=a \varrho_{-1,} \zeta^{1-b} X^{\eta-a}(\xi-\eta X) \text {. }
$$

This equation conld only reduce to an identity for $a=2$, but even then we get a contradiction because $\bar{\eta} / \xi$ is not a root of unity. The remaining case $u= \pm 1, v=-r$ is dealt with similarly. This completes the proof of Theorem 2 .

5 - Acta Arithmettica $x \times x v x_{12}$ 
[1] J. H. Conway and A. J. Jones, Trigonometric diophantine equalions (On vanishing sums of roots of unity), Acta Arith. 30 (1976), pp. 229-240.

2] V. E nnola, $A$ note on a cyclotomis clioptantine equation, ibid. 28 (1975), pp. 157-159.

[2] V.Ennola, A mote on on the solutions of diophantine equations in units, ibid. 30 ]. F. H. Grossman, On th

4] K. Györy, Sur une classe des corps do nombres algébriques et ses applications. Publ. Math. (Debrecen) 22 (1975), pp, 1.51.-1.75.

5] - Representation des nombres enliers par des formes binaires, to appowr in I’ubl. Math. (Debrecon)

[B] ] P. Postnilova and A. Solinzal, Primitivo divisors of the oxpression $a^{n}-b^{n}$ in algebrais number fields, Mat. Sbornik 75 (1968), pp. 1.71--177 (in Rusaian).

7] A. Schinzel, Primitive divisors of the expression $A^{n}-B^{n}$ in algebraio number fields, J. Roine Angew. Matl. 268/209 (1.974), pp. 27-33

Received on 30.7. 1977

\section{Rosser's sieve}

by

HJiNRYK IWANIEO (Warsaw-Pisa)

1. Introduction. Statement of the results. Let there be given a finite integer sequence $\mathscr{A}$ and a sequence $P$ of primes. A basic problem of the sieve is to estimate, for any real number $z \geqslant 2$ the sum (sifting function)

$$
S(\mathscr{A}, P, \approx)=\sum_{\substack{a \in \mathscr{\alpha} \\(a, P(z))=1}} 1
$$

where $P(z)=\prod_{p<z_{1} \in \mathcal{P}} p$. The sequence $\mathscr{A}$ can bo almost axbitrary. The only knowledge we need about $\mathscr{A}$ is a good approximation formula (in an average sense) for the number of those elements from $\mathscr{A}$ which aro divisible by the squarefree number $d \mid P(z)$;

$$
\mathscr{A}_{d}=\{a \in \mathscr{A} ; a \equiv 0(\bmod d)\} .
$$

Wo assume that $\left|\mathscr{A}_{d}\right|$ may be written in the form

$$
\left|\mathscr{A}_{d}\right|=\frac{\omega(d)}{d} X+R(\mathscr{A}, d)
$$

where $\frac{\omega(d)}{d} X$ is considered as a main term and $R(\mathscr{A}, d)$ is an error term. The arithmetic function $\omega(d)$ is multiplicative and for each prime number $p \in P$ it satisfies

$$
0<\omega(p)<p
$$

Since wo need the formula (1.1) only for $d \mid P(z)$ we are free to define $\omega(p)$ $=0$ for $p \notin P$.

Our next assumption is about dimension. There exists a parameter $x \geqslant 0$ (dimension) and a constant $K \geqslant 2$ such that for all $z>w \geqslant 2$ we have

$$
\prod_{w<x<z}\left(1-\frac{\omega(p)}{p}\right)^{-1}<\left(\frac{\log z}{\log w}\right)^{*}\left(1+\frac{K}{\log w}\right)
$$

